\title{
Self in motion: sensorimotor and cognitive mechanisms in gait agency
}

\author{
O. A. Kannape ${ }^{1}$ and O. Blanke $e^{1,2,3}$ \\ ${ }^{1}$ Laboratory of Cognitive Neuroscience, Brain Mind Institute, School of Life Sciences, École Polytechnique Fédérale de \\ Lausanne, Lausanne, Switzerland; ${ }^{2}$ Center for Neuroprosthetics, School of Life Sciences, École Polytechnique Fédérale de \\ Lausanne, Lausanne, Switzerland; ${ }^{3}$ Department of Neurology, University Hospital, Geneva, Switzerland
}

Submitted 4 December 2012; accepted in final form 1 July 2013

\begin{abstract}
Kannape OA, Blanke O. Self in motion: sensorimotor and cognitive mechanisms in gait agency. J Neurophysiol 110: 1837-1847, 2013. First published July 3, 2013; doi:10.1152/jn.01042.2012.— Acting in our environment and experiencing ourselves as conscious agents are fundamental aspects of human selfhood. While large advances have been made with respect to understanding human sensorimotor control from an engineering approach, knowledge about its interaction with cognition and the conscious experience of movement (agency) is still sparse, especially for locomotion. We investigated these relationships by using life-size visual feedback of participants' ongoing locomotion, thereby extending agency research previously limited to goal-directed upper limb movements to continuous movements of the entire body. By introducing temporal delays and cognitive loading we were able to demonstrate distinct effects of bottom-up visuomotor conflicts as well as top-down cognitive loading on the conscious experience of locomotion (gait agency) and gait movements. While gait agency depended on the spatial and temporal congruency of the avatar feedback, gait movements were solely driven by its temporal characteristics as participants nonconsciously attempted to synchronize their gait with their avatar's gait. Furthermore, gait synchronization was suppressed by cognitive loading across all tested delays, whereas gait agency was only affected for selective temporal delays that depended on the participant's step cycle. Extending data from upper limb agency and auditory gait agency, our results are compatible with effector-independent and supramodal control of agency; they show that both mechanisms are dissociated from automated sensorimotor control and that cognitive loading further enhances this dissociation.
\end{abstract}

agency; self; human locomotion; dual task; cognition

WHENEVER WE MOVE, or observe someone else moving, we automatically attribute this action to its corresponding agent, effectively separating ourselves from our environment (Jeannerod 2003) but also from other agents (Jeannerod 2006). In many cases this distinction is quite apparent, as we can see the body part of an agent move with respect to his or her body, whereas the movement of our own limbs is contingent with our visuospatial perspective (David et al. 2006) and aligned with respect to our own central reference frame (see Kannape and Blanke 2012); in some cases, the movement we observe simply does not correspond to our intended movement (Daprati et al. 1997; van den Bos and Jeannerod 2002). However, there are situations in which multiple agents, such as surgeons during a medical procedure, perform similar movements in parallel or rapid succession. In this case we may not be able to correctly attribute the action to the correct agent based on salient features such as visuospatial perspective or motor-intention. Instead, it

\footnotetext{
Address for reprint requests and other correspondence: O. Blanke, Laboratory of Cognitive Neuroscience, Brain Mind Institute, Station 19, École Polytechnique Fédérale de Lausanne (EPFL), 1015 Lausanne, Switzerland (e-mail: olaf.blanke@epfl.ch).
}

is understood that the sense of agency relies on a central monitoring framework (Frith and Done 1989), in essence a comparison of internal representations and predictions about our movements (using the efference copy) with the feedback we continuously receive about those movements (reafferent signal, but also with our intended or desired state; see Blakemore et al. 2002). The central monitoring hypothesis extends the understanding that our brains use internal models for sensorimotor control in the so-called observer framework (Wolpert et al. 1995), which efficiently optimizes motor control without the need for cognitive supervision. This framework is based on physiological mechanisms of corollary discharge as introduced by Sperry (1950), von Holst and Mittelstaedt (1950), and, previously, Helmholtz (1866).

While internal models convincingly account for automatic sensorimotor behavior (Todorov and Jordan 2002), the extended central monitoring framework has been criticized as falling short of explaining specific aspects of the sense of agency. In particular the distinction between a feeling of agency, closely tied to sensorimotor control, and a judgment of agency, a post hoc evaluation of one's actions additionally based on salient features such as task completion, is incomplete (Synofzik et al. 2008b), as is the attribution of covert actions (Jeannerod and Pacherie 2004). One reason is that, although the sense of agency has recently been intensively studied, it is quite difficult to target specific aspects of agency processing (see Haggard and Chambon 2012). Hence the vast majority of agency studies have used goal-directed paradigms in which spatiotemporal mismatches were introduced between participants' upper limb movements and the feedback they received. Participants evaluated movements of their fingers (Knoblich and Repp 2009; Repp 2005, 2006; Repp and Knoblich 2007), hands (Daprati et al. 1997; Daprati and Sirigu 2002; van den Bos and Jeannerod 2002), and arms ( Knoblich et al. 2004; Nielsen 1963; Synofzik et al. 2006) with respect to a specific objective and after actions had been completed. This leaves open a number of questions.

First, does the sense of agency differ between movements of individual body parts and actions involving the entire body? As the aforementioned publications indicate, research into the sense of agency has mainly focused on actions of fingers, hands, and arms. Whereas control during such reaching and pointing movements has been linked to parietal regions (Castiello 2005; Kandel 2012), locomotion is highly automated and basic stepping mechanisms may function in the absence of cortical centers (Armstrong 1988; Grillner and Wallen 1985). Thus important locomotor regions have been described in the cerebellum and midbrain (Jahn et al. 2008b; Shik and Orlovsky 1976) in addition to a large body of evidence underlining the 
contributions of reflex mechanisms and central pattern generators in cat (Perret and Cabelguen 1980) and, more recently, human (Dietz et al. 1994) spinal cord. Along these lines, purely reflexive neuromuscular models have been proposed that can account for important adaptive behavior during locomotion without the involvement of feedforward neural control (Geyer and Herr 2010; Markowitz et al. 2011). This suggests that cortical systems of motor control and agency for locomotion may differ from those described for upper limb movements, and it is questionable whether or not the details of such automated movements are available for conscious monitoring or introspection at all.

Second, does the sense of agency differ for continuous movements? One aspect of locomotion is that it is continuous and most often not immediately goal-directed (see Kannape and Blanke 2012). We have recently shown that, in a goaldirected paradigm, the limits of agency for locomotion are comparable to those of upper limb movements (Kannape et al. 2010). This may reflect that at least the judgment of agency can be described by an effector-independent mechanism that predominantly depends on salient cues such as the successful completion of a given task. Investigating continuous walking allows us to employ a paradigm that differs from previous experimental approaches, in which movements followed a specific task or movement instructions (Daprati et al. 1997; van den Bos and Jeannerod 2002) and were mostly directed toward an object (or position) in peripersonal space (i.e., goal-directed; Fourneret and Jeannerod 1998; Franck et al. 2001; Synofzik et al. 2006). In the paradigm we propose below, agency attribution may therefore not be based on a post hoc evaluation of the movement and its goal, i.e., a judgment of agency, as there is no direct immediate outcome to evaluate, such as reaching a target or the press of a button. Instead, it has to be based on the sensorimotor control loop and constantly evaluated against the reafferent sensory information. Leube and colleagues (2003a, 2003b) investigated agency for continuous hand movements

A

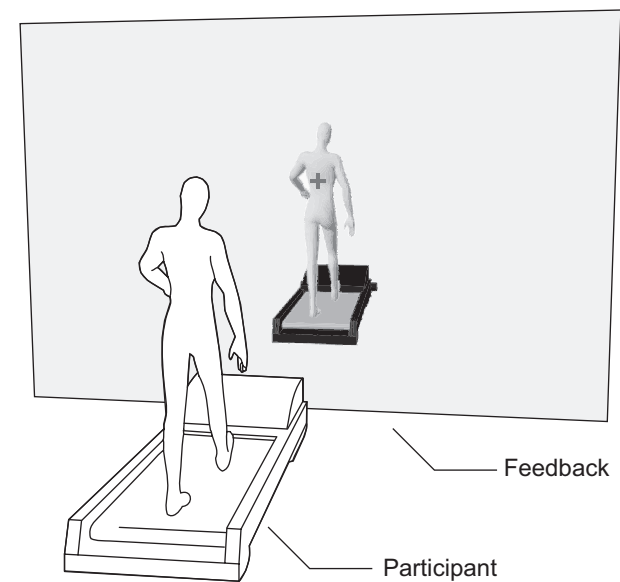

and proposed that processing of the underlying efference copy adapts to the motor and sensory areas specific to the investigated movement. This suggests that the sense of agency for continuous full-body movements may be similar to that described for hand agency.

Third, by combining locomotion with a secondary task we investigated cognitive contributions to the sense of agency and how gait movement, gait agency, and cognition interact. On one hand, much research has been carried out on the effects of cognitive loading and gait control (Woollacott and ShumwayCook 2002; Yogev-Seligmann et al. 2008). Thus humans may perform perceptual, motor, and cognitive tasks while walking, but their performance in these tasks may decline as a consequence. On the other hand, no data exist on the influence of cognition or limiting cognitive resources on agency processing. The fact that agency processing is intertwined with the sensorimotor control loop, which is impaired by the introduction of a second task, implies that agency processing should also deteriorate in such a dual-tasking paradigm. By investigating gait agency under cognitive load we can add an important insight into agency mechanisms in general.

Here we investigated sensorimotor control of full-body movements and its accompanying sense of agency by adapting a classical paradigm for upper limb agency to locomotion. Participants continuously walked on a treadmill and watched an individually mapped, life-size virtual body perform their own movements either in real time or with a randomized additional delay between $25 \mathrm{~ms}$ and 1,350 ms (see Fig. 1). In each trial the participants received visual feedback of their gait for $3 \mathrm{~s}$, after which they were asked to judge whether the movement that the virtual body had performed was the movement they had just made (Franck et al. 2001; Kannape et al. 2010). In another experimental block, counterbalanced design, participants performed the same experiment while executing the articulated serial sevens subtraction task. We predicted that, as for hand agency, gait agency would strongly depend on the

B

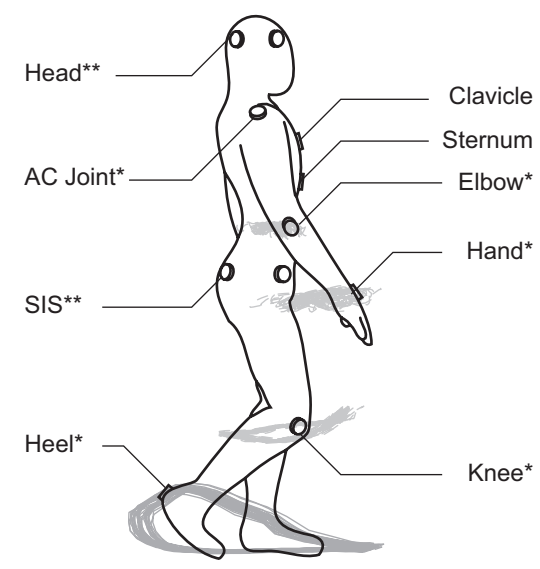

Fig. 1. A: experimental setup. Participants walked on a treadmill while their movements were captured by way of an optical motion capture system. These movements were mapped onto an individually adapted life-size avatar and played back on a rear-projection screen placed $3 \mathrm{~m}$ in front of the participant. Each trial consisted of a 2-s preparatory period in which only the fixation cross was visible, followed by $3 \mathrm{~s}$ of gait feedback during which both the avatar and the fixation cross were visible (see Supplemental Video S1). Feedback was either presented in real time (75 ms) or randomly delayed by up to 1,350 ms. Participants judged by pressing a button whether the movement they saw on the screen exactly corresponded to the movement they had just performed. $B$ : marker placements. Participants wore 20 infrared markers: 1 each on the sternoclavicular joint and the lower sternum; *2 on left-right heel, lateral knee and elbow, dorsal hand and acromioclavicular (AC) joint; **4 on left-right, anterior-posterior superior iliac spine (SIS) and head. Characteristic movement patterns are shown for the elbow, hand, knee, and heel markers. Stride time data were extracted from the latter. 
temporal mismatch of the feedback; furthermore, agency judgments may be high for feedback delayed by an entire step cycle as the current and a previous feedback step cycle coincide. We predicted that gait agency would be affected by taxing cognitive resources, as the introduction of a secondary task has been shown to interfere with the cortical control of locomotion.

\section{MATERIALS AND METHODS}

Fifteen healthy participants with normal or corrected to normal vision volunteered for the study ( 8 women, 7 men; age $=23 \pm 6 \mathrm{yr}$, height $=173 \pm 11 \mathrm{~cm}$, weight $=65 \pm 13 \mathrm{~kg}$ ). The study was conducted according to the principles expressed in the Declaration of Helsinki and was reviewed and approved by the local ethics committee (University Hospital Lausanne, Switzerland). All participants provided written informed consent for the collection of data and subsequent analysis. The study, including participant information, setup, and breaks, lasted for $4 \mathrm{~h}$, and participants were remunerated 20 $\mathrm{CHF} / \mathrm{h}$.

\section{Treadmill}

Participants continuously walked on a treadmill (U.N.O. X-Trail 2.0, Beny Sports, Nürnberg, Germany) for an entire experimental block. The console and right handlebar were detached from the treadmill so that participants had an unimpeded view of the visual feedback and could easily exit from the treadmill to one side (in which case the experiment was automatically paused). The left handlebar (not shown in Fig. 1) was reinforced and extended in order to provide assistance. Participants were instructed to swing their arms as during over-ground walking and only use the handlebar if they lost balance.

\section{Motion Capture and Feedback}

The participants' movements were tracked and recorded by an active optical motion capture system (20 infrared markers, ReActor2, Ascension Technology, Burlington, VT) at a sampling frequency of $30 \mathrm{~Hz}$ (see also Kannape et al. 2010). The marker positions, as indicated in Fig. $1 B$, were one each on the sternoclavicular joint and the lower sternum, two each (left, right) on the heel, lateral knee, lateral elbow, dorsum of the hand, and acromioclavicular (AC) joint, and four each on the left-right, anterior-posterior superior iliac spine (SIS) and the head. A commercial real-time productivity suite was used (Autodesk MotionBuilder, San Rafael, CA) for three-dimensional character animation. This software facilitated mapping of a virtual character onto the set of optical markers worn by the participant while integrating natural biped kinematics. A customized skeleton was thus fitted for each participant to align the joints of the virtual character with the participant's actual joints. Participants received life-size visual feedback of their movements by way of a $3.20 \times$ 2.35-m back-projection screen (width $\times$ height, 1,280 $\times 1,024$ pixels, $60 \mathrm{~Hz}$ ), with the screen itself forming the back wall of the tracking arena (JVC DLA-SX21 projector, JVC U.S.A., Wayne, NJ). The distance between the participant and the projection screen was $\sim 3 \mathrm{~m}$. The overall delay of the system, including data acquisition, character animation, and visual presentation, was $\sim 75 \mathrm{~ms}$. Participants started the experiment and gave their agency judgments with a wireless gamepad (XBOX 360 controller, Microsoft, Redmond, WA), which they held in the right hand.

\section{Paradigm}

Before the experiment the treadmill speed was adapted individually ( $\mu=1.07 \mathrm{~m} / \mathrm{s}, \sigma=0.07$ ) so that all participants would have a similar stride time throughout the experiment ( $\mu=1.31 \mathrm{~s} ; \sigma=0.05$ ). In each trial (144 per condition), participants watched an individually mapped, life-size virtual body perform their own movements either in "real time" (with an intrinsic delay of $75 \mathrm{~ms}$ ) or with a randomized additional delay $(25,50,75,100,150,225,300,450,600,750,900$, $1,050,1,200,1,125,1,275,1,350 \mathrm{~ms} ; 8$ trials per delay, 16 real-time trials). A schematic of the setup is illustrated in Fig. 1. Each trial began with only the fixation cross and treadmill shown on the screen ( $2 \mathrm{~s})$. Subsequently, participants received visual feedback of their gait for $3 \mathrm{~s}$, after which they were asked via a pop-up text box: "Did the movement shown on the screen correspond to the movement you just performed?" (self-attribution; Franck et al. 2001; Kannape et al. 2010; see Supplemental Video S1). ${ }^{1}$ By pressing a button on the joypad participants indicated whether the movement that the virtual body had performed was the movement they had just made. Participants wore closed headphones that played white noise to remove auditory feedback from their footfalls. The fixation cross remained visible during the trials to avoid participants focusing on the movement of individual extremities. Participants continuously walked for the duration of the entire experimental block, including the response phase at the end of each trial.

We stress that the present paradigm does not allow for gait adaptation, as feedback only lasted for $3 \mathrm{~s}$, five steps on average, and as delays were randomized across trials. Furthermore, participants were not required to adapt their movements in order to maintain gait or balance [as, e.g., is the case for walking on a split-belt treadmill (Jensen et al. 1998; Torres-Oviedo and Bastian 2010) or a circular treadmill (Gordon et al. 1995) or with unilateral weighting of a leg (Noble and Prentice 2006)]. In line with previous literature, we deliberately chose to exclusively use feedback generated by the participants themselves. This ensured that agency attributions depended on the chosen independent variable, the temporal mismatch. Introducing movements from other actors may introduce confounding factors and result in a self-other distinction paradigm, as humans are particularly sensitive to biological movement and can readily distinguish between their own, a familiar, or another person's movement (Blake and Shiffrar 2007).

\section{Cognitive Load}

Introducing a secondary, cognitive task during locomotion has been shown to alter body posture and gait characteristics and to lead to freezing or falls (for comprehensive reviews see Woollacott and Shumway-Cook 2002 and Yogev-Seligmann et al. 2008). In particular, the serial sevens subtraction task induces gait changes such as a decrease in velocity [in young healthy participants and patient populations (Springer et al. 2006)], an increase in stride length and stride time [in healthy elderly participants (van Iersel et al. 2007) and patients], and increased gait variability [in neurological patients with Parkinson's disease (Hausdorff et al. 2003; Yogev et al. 2005)]. We therefore chose the serial sevens subtraction task to investigate the influence of taxing cognitive resources on gait agency, gait movements, and their interaction. Participants thus performed two blocks of the study, which were counterbalanced across participants, one with and one without the subtraction task.

Arguably, participants carry out three simultaneous tasks in this condition, namely, walking, counting, and performing the agency task. As we are particularly interested in possible effects and interaction due to the introduction of the serial sevens subtraction task, we will refer to the two conditions as single task (ST) and dual task (DT).

\section{Data Analysis}

Agency judgments and gait characteristics were recorded throughout the entire study and processed off-line with the $\mathrm{R}$ language and

\footnotetext{
${ }^{1}$ Supplemental Material for this article is available online at the Journal website.
} 
environment for statistical computing ( $\mathrm{R}$ Development Core Team 2011).

Stride time extraction. Stride time data were determined separately for each leg with the corresponding heel markers, with the SIS markers serving as reference. The timestamps of the motion capture system were synchronized to the onset/offset of the visual feedback (1-ms timestamps) so that only the $3 \mathrm{~s}$ of movement data recorded during the visual feedback were used in the analysis for each trial. Only complete cycles (for each leg) were included in the average for each trial, and cycles shorter than 1,000 ms or longer than 2,000 ms were rejected.

Usually a gait cycle is defined to begin with the heel strike of one foot and end at the subsequent heel strike of the same foot. To maximize the amount of information in the 3-s trials we used the first data sample as the start point for all gait cycles in a given trial (as opposed to waiting for the first heel strike to occur). In detail, we first calculated the mean position of the four SIS markers to calculate a dynamic reference point. Using this dynamic position as a reference allows us to include the possible translation of the full body in the $z$-plane. If the participant speeds up or slows down, a difference in his absolute position would not affect the calculated gait cycle. Individual step cycles were then calculated with the distance in the $z$-plane between the initial position of the heel marker and the initial position of the reference SIS markers and extracting the two data points closest to the second crossing of this initial position. The foot thus passed once in the swing and once in the stance phase (or vice versa). If the first data point was the minimum or maximum in a trial, the cycle started when the heel crossed the $z$-coordinate of the reference markers for the first time. To improve spatiotemporal accuracy of the data we calculated the exact point of intersection based on the coordinates and timestamps of the two adjacent motion capture data points.

Using this analysis, we calculated the stride time for each leg separately as well as for each temporal delay. Furthermore, we determined the standard deviation of the stride time and the coefficient of variation $(\mathrm{CV})$, i.e., the ratio of the standard deviation over the absolute mean. The same values (mean, standard deviation, and CV) were also calculated for the stride length. This allowed us to analyze gait data separately for all delays and also check for potential gait asymmetries.

Normalized delay. The amount of mismatch between the visual feedback (avatar's gait) and the sensorimotor feedback (participant's gait) directly depends on the relation between the introduced temporal delay and the concurrent stride time, i.e., feedback delayed by 1,200 ms will appear synchronous to a participant with a stride time of 1,200 $\mathrm{ms}$ but not to a participant with a stride time of $1,400 \mathrm{~ms}$. To compare the effect of feedback delay on stride time across delays and across participants, we therefore expressed the independent variable (Delay) in terms of the fraction of the corresponding stride time. For a person walking with a stride time of 1,200 ms, a delay of $600 \mathrm{~ms}(1,200 \mathrm{~ms})$ would hence correspond to half a step cycle (1 step cycle) delay.

Agency thresholds. Temporal thresholds were determined by fitting a cumulative Gaussian to the agency responses for trials with 0-ms to 600-ms delays with the published psignifit toolbox (Wichmann and Hill 2001a, 2001b) for MATLAB (MathWorks, Natick, MA). This toolbox enforces bootstrapping algorithms and weighs the individual data points based on the number of valid trials per stimulus intensity. All thresholds reported here reflect the $50 \%$ point of subjective equality.

\section{Statistical Analysis}

We set up a $2 \times 9$ factorial design with factors Task (single task, dual task) and Delay (9 different delays; see above). Repeatedmeasures analyses of variance (ANOVAs) and post hoc comparisons were performed in Statistica (StatSoft, Tulsa, OK). The temporal thresholds and the width of the psychometric fit as well as the root-mean-square (RMS) values extracted from the stride time were compared with Student's $t$-test, two-tailed and paired. The linear and nonlinear models for the stride time data were fitted and compared in $\mathrm{R}$ with the Aikake and Bayes Information Criteria (AIC and BIC, respectively) (Burnham 2004; Rodríguez 2005).

All participants were healthy young adults, confident in and familiar with treadmill walking and therefore stable. They were instructed to walk steadily, swinging their arms at their sides as they would during free walking. Nonetheless, we asked participants to step down from the treadmill should they lose their balance and not answer the agency question. This occurred less than once per participant and block on average. Trials were automatically rejected from the analysis based on the following criteria (and in this order): 1) participants took longer than $10 \mathrm{~s}$ to respond, e.g., after losing balance and stepping off the treadmill; 2) the response time deviated $>3$ SDs from the mean; 3) a gait cycle had a stride time outside the range of 1,000-2,000 ms; 4) a gait cycle deviated $>3$ SDs from the mean. If a trial was rejected because of a corrupted or missing agency response, the entire trial (both agency response and gait data) was removed from the analysis. Gait cycles were rejected individually. On average 3.4 of 144 trials (per participant and block) were rejected because of deviations in response times, including 0.4 trials (per participant and block) rejected because participants exceeded the maximum of response time of $10 \mathrm{~s}$.

\section{RESULTS}

We hypothesized that gait agency would depend on the spatiotemporal congruency of the feedback and decrease linearly with longer delays as has been previously shown for upper limb movements (e.g., Franck et al. 2001). Owing to the cyclic nature of locomotion, we also hypothesized that gait agency could be misled by feedback delayed by an entire gait cycle. With respect to the taxing of cognitive resources, we hypothesized that cognitive loading would alter gait agency, as both processes rely on higher-level (likely cortical) mechanisms.

Our hypothesis for the effects of Delay and Task on gait characteristics differed from those for gait agency. Previous research on the sense of agency has illustrated that participants adapted their motor performance to the sensorimotor mismatch in the feedback, even if they had not become aware of such a mismatch (Fourneret and Jeannerod 1998; Kannape et al. 2010). However, this was intrinsically tied to the design of the experiments, as participants tried to complete a goal-directed task (Kannape and Blanke 2012). In the present paradigm, participants continuously walked on a treadmill, at fixed speed. We therefore did not predict a delay-dependent effect on the gait characteristics. Furthermore, dual-task paradigms have consistently been shown to lower walking speed while increasing gait variability (Woollacott and Shumway-Cook 2002). We therefore hypothesized that locomotion would generally be impaired by taxing cognitive resources, independent of delay.

\section{Single-Task Condition}

Gait agency. Our data reveal three main findings for gait agency, which depended on the delay between the participant's actual movement and the avatar's movement [repeated-measures ANOVA, main effect of Delay: $F(8,112)=60.054, P<$ $0.001]$. First, participants judged the vast majority of nondelayed (ND) feedback trials as self-generated (self-attribution: $\mu=84.3 \pm 3.6 \%$ SE; most likely agency did not reach $100 \%$ because of the intrinsic delay of $75 \mathrm{~ms}$ of the optical tracking and animation system). Second, as illustrated in Fig. 2A, gait 

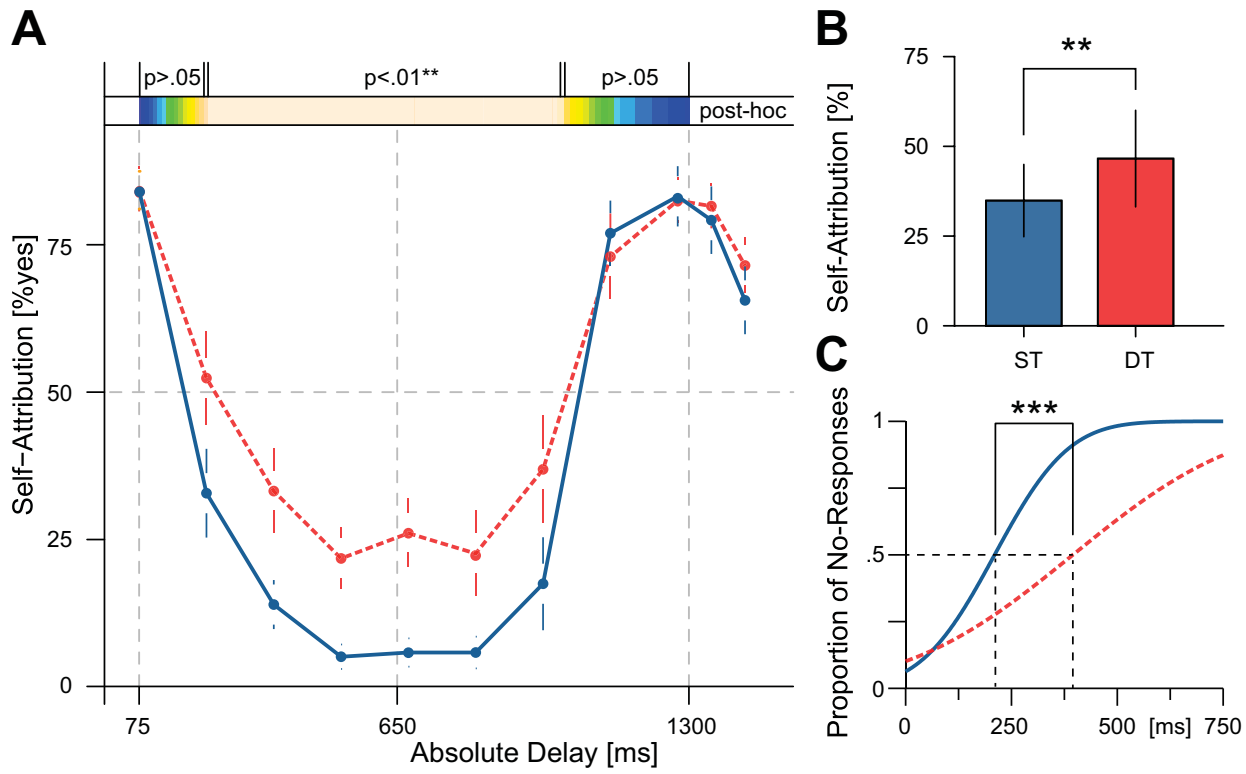

Fig. 2. Gait agency. A: agency ratings as a function of feedback delay: In the single-task (ST) condition (solid blue line) participants reported agency for the majority of real-time trials. Self-attribution decreased with increasing delays and reached a minimum after $525 \mathrm{~ms}$. A delay of $675 \mathrm{~ms}$ corresponded to half a step cycle and was easiest for participants to discriminate as the avatar appeared to be left-right reversed. Increasing the delay past $1,125 \mathrm{~ms}$ led to a high percentage of trials being again self-attributed, indicating that participants could not distinguish between their current and a previous step cycle. Agency judgments followed a similar pattern in the dual-task (DT) condition (dotted red line). However, cognitive loading selectively impaired judgments for delays between $225 \mathrm{~ms}$ and $1,125 \mathrm{~ms}$, leading to a significant increase in erroneously self-attributed trials. Agency was not affected for real-time trials and trials delayed by a full step cycle. $B$ : cognitive loading led to a significant increase in self-attributed trials. However, this was only true for trials with delays between 175 ms and $975 \mathrm{~ms}$, as indicated by the individual post hoc comparisons (A, top). Agency ratings were identical for real-time trials and trials delayed by a full step cycle. C: temporal thresholds were determined by fitting a cumulative Gaussian (cf. Data Analysis). Thresholds increased from $210 \mathrm{~ms}$ to almost $395 \mathrm{~ms}$ under cognitive load. At the same time, the slope of the psychometric fit over the threshold region significantly decreased, signifying that participants were less certain about their responses. All error bars show SE. $* * P<0.01, * * * P<0.001$.

agency decreased with increasing delays so that participants stopped judging the majority of movements to be self-generated for delays of $>210 \mathrm{~ms}(50 \%$ point of subjective ambiguity: $210 \pm 26 \mathrm{~ms}$, including 75-ms intrinsic delay; see Fig. 2C). Gait agency continuously decreased with increasing delays and was lowest for delays ranging from $375 \mathrm{~ms}$ to $975 \mathrm{~ms}$, where it dropped to an average of $9.7 \%( \pm 4.5 \%)$. For these trials the actual movement and the visual feedback were on average separated by half a step-cycle delay (HD) and were therefore maximally out of phase so that the visual feedback (avatar) appeared left-right reversed with respect to the ongoing movements of the participant. Third, by introducing delays that were as long as the participants' step cycles (ranging from $1,191 \mathrm{~ms}$ to $1,432 \mathrm{~ms}$ ) we could show that participants were unable to distinguish between their actual full-body movement and resynchronized visual feedback: gait agency for trials with a full step-cycle delay (FD) was high and identical to gait agency for ND trials $(83.2 \% \pm 5.7 \%$, planned comparison between ND and FD trials: $P=0.87$ ).

Gait movements. Concerning gait parameters, we found that participants systematically modulated their stride time as a function of the temporal delay of the visual feedback they received [repeated-measures ANOVA, main effect of Delay $F(8,104)=6.2362, P<0.001]$. This was found even though treadmill speed was fixed and participants therefore had to adjust their gait cycle by changing both stride time and stride length. Participants took slower than average steps when visual feedback was synchronous with their ongoing movement, and this was the case for ND trials and maximally delayed but resynchronized visual feedback trials (FD feedback trials; Fig. $3 A$ ); participants took faster than average steps (i.e., shorter step cycles) for feedback that was temporally and spatially out of phase ( $1 / 4$ and $3 / 4$ delay). We also found that step cycles were slower for trials with a delay of half a step cycle (HD). These trials were temporally but not spatially congruent. The stride time $\mathrm{CV}$, an indicator of gait variability, reversely reflected the sinusoidal modulation observed for the stride time deviations: CV was lowest for rhythmically synchronous feedback (ND, HD, and FD) and highest for in-between delays with approximately a $1 / 4$ or $3 / 4$ step cycle delay, signifying that participants' gait varied most when feedback was neither temporally nor spatially congruent $[F(8,104)=2.0473, P=0.048]$.

To summarize, we report that participants adjusted their gait (stride time) to the temporal synchrony of the visual feedback. Importantly, these changes showed a dependence on the step cycle different from the changes observed for gait agency and were not required by the task or due to external, physical constraints imposed on the participants.

\section{Dual-Task Condition}

Gait agency under cognitive load. For gait agency, we found a main effect of Task $[F(1,14)=21.608, P=0.00038$; Fig. $2 B$ ] caused by significantly higher erroneous self-attribution in the DT than the ST condition (36\% ST vs. 48\% DT; difference of $12 \%$ ). The perceptual thresholds accordingly increased from $210 \pm 26 \mathrm{~ms}$ in the ST condition to $395 \pm 55 \mathrm{~ms}(P<0.001$, $t$-test) in the DT condition. Furthermore, the width of the individual sigmoid fits significantly increased in the DT condition $(P=0.006, t$-test $)$, signifying that participants were less certain of their responses (see Fig. 2C). As for the ST condi- 

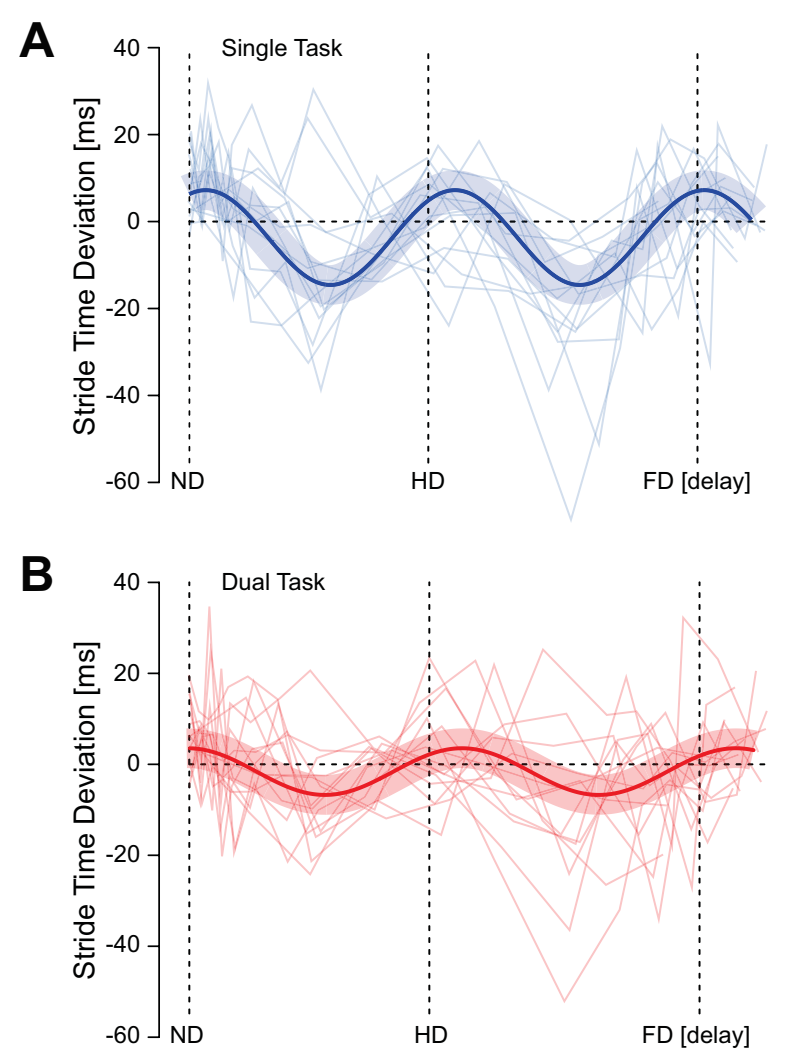

Fig. 3. Gait movements. A: independent of the task, participants changed their stride time ( $y$-axis) as a function of the normalized delay of the visual feedback ( $x$-axis). HD delay corresponds to a mismatch of a single step and FD delay to a full step cycle. Participants automatically adjusted their gait and took slower than average steps for temporally synchronous feedback [nondelayed (ND), FD trials], even when it appeared left-right reversed (HD trials). They took faster than average steps for feedback that was out of phase as illustrated by the sinusoid fit. $B$ : stride time data under cognitive load: the automatic gait synchronization observed in the ST condition was significantly suppressed across all delays, suggesting that the secondary task impeded the automatic stride time adjustment.

tion, gait agency in the DT condition did not differ between $\mathrm{ND}$ and FD trials (planned comparison $P=1$ ) and gait agency for these synchronous feedback trials did not differ between the ST and DT conditions (planned comparisons, all $P=1$ ). Response times increased from $792 \pm 87$ to $1,196 \pm 116 \mathrm{~ms}$ (mean $\pm \mathrm{SD}, P<0.001$; Table 1). However, participants were not instructed to respond as quickly as possible, as we were interested in accurate agency responses and correct performance in the secondary task.

Statistical analysis also revealed a significant interaction between the two factors Task and Delay $[F(8,112)=3.1156$, $P=0.00325]$. Self-attribution in the DT vs. ST conditions only increased for delays between 225 and $975 \mathrm{~ms}$, explaining the significant interaction (13\% ST vs. $32 \%$ DT; difference of $19 \%)$. The increase in erroneous self-attribution that we observed during cognitive loading was caused by these specific delays and was absent for ND and FD feedback trials. The significant interaction between DT and delay rules out that the increase in self-attribution between a participant's ongoing movement and the avatar's movement when performing the serial sevens subtraction task while walking was a result of general task difficulty or a general trend of our participants to always overattribute movements of the avatar to themselves during the DT condition. If the former were true, gait agency would approach $50 \%$ (chance) across all delays, whereas for the latter, gait agency would increase for all delays. This was not the case, as gait agency was not affected for ND and FD trials.

In summary, gait agency strongly depended on the temporal delay of the feedback in both experimental conditions. Cognitive loading did not impair agency judgments for temporally and spatially congruent feedback (ND and FD trials). However, our participants' judgments were significantly affected under cognitive loading for delays between 225 and $975 \mathrm{~ms}$ as reflected in the greatly increased temporal thresholds.

Gait movements under cognitive load. Corresponding to previous studies and as predicted, there was a main effect of Delay over both conditions $[F(8,104)=5.7687, P<0.001]$ but no main effect of Task $[F(1,13)=0.14520, P>0.7]$ and no interaction on the overall stride time using the absolute delays $[F(8,104)=1.2616, P>0.2$; cf. Table 1]. As observed in the ST condition, stride time still significantly depended on the temporal delay, but as illustrated in Fig. 3, $A$ and $B$, the modulation was weaker than in the ST condition. The systematic change of slower step cycles during ND, HD, and FD trials persisted but, as indicated in the following, was significantly dampened by cognitive loading. Importantly, unlike gait agency, which was affected differentially, these changes in stride time were altered uniformly across all tested delays.

While the parametric statistics already confirmed a significant overall effect of temporal delay on gait movements, we fitted a sinusoidal model to the normalized gait data. The sinusoidal models confirmed the regularity of the stride time modulation (see Fig. 3A) and emphasized the effect of cognitive loading. In the ST condition the four-parameter sine model explained $26 \%$ of variance with a residual standard error of 12.69 on 210 degrees of freedom (DoF) compared with $4 \%$ variance accounted for and a residual standard error of 14.34 on $212 \mathrm{DoF}$ for a linear fit (adjusted $r^{2}=0.043$ ). We further used the BIC and AIC, which account for the different numbers of parameters in the models, to compare the goodness of fit for the linear and nonlinear models. This analysis yielded a comparative Bayes factor of $2.9 \times 10^{9}$ and an AIC factor of $8.7 \times$ $10^{10}$, indicating that the sinusoid presents a significantly better fit than the linear model (Burnham 2004; Rodríguez 2005). While a four-parameter sinusoid model also yielded a smaller residual standard error than a linear model in the DT condition (12.2 on 210 DoF, 12.57 on 212 DoF), both models accounted for less variance ( $8 \%$ and $1 \%$, respectively). Furthermore, the difference in the goodness of fit between the two models was significantly smaller (Bayes factor $=7$, AIC factor $=208$ ).

The amplitude of the sine model's fit to the stride time data reflects the extent to which participants adjusted their gait cycle. This value was halved going from the ST to the DT condition. We therefore calculated the RMS stride time deviation to describe the effective deviations in stride time. We found that the RMS deviation did not differ significantly between the two conditions $(P>0.5, t$-test $)$ despite the large change in amplitude. This indicates that participants' stride time changed to a similar extent as in the ST condition, but no longer in a systematic fashion. There was a strong, positive correlation between RMS deviation for ST and DT across participants $\left(r^{2}=0.447, P=0.012\right)$. Taken together, these results confirm that the secondary task suppressed the auto- 
Table 1. Response time and gait characteristics

\begin{tabular}{|c|c|c|c|c|c|c|}
\hline & Condition & Mean & SD & \multicolumn{2}{|c|}{ Main Effect } & Interaction $\mathrm{T} \times \mathrm{D}$ \\
\hline Response time, ms & ST & 792 & 87 & $P<0.001 * * *$ & $P=0.042 *$ & $P=0.66$ \\
\hline & DT & 1,196 & 116 & & & \\
\hline \multirow[t]{2}{*}{ Stride time, $\mathrm{ms}$} & ST & 1,304 & 13 & $P=0.71$ & $P<0.001 * * *$ & $P=0.27$ \\
\hline & DT & 1,306 & 13 & & & \\
\hline Stride time CV & ST & 2.4 & 0.19 & $P=0.61$ & $P=0.048^{*}$ & $P=0.82$ \\
\hline & DT & 74.6 & 1.9 & & & \\
\hline \multirow[t]{2}{*}{ Step length CV } & ST & 9.2 & 1.1 & $P=0.040^{*}$ & $P=0.79$ & $P=0.43$ \\
\hline & DT & 10.8 & 1.3 & & & \\
\hline \multirow[t]{2}{*}{ Step width, cm } & ST & 8.9 & 0.8 & $P=0.08$ & $P=0.07$ & $P=0.85$ \\
\hline & DT & 9.2 & 0.9 & & & \\
\hline \multirow[t]{2}{*}{ Lateral hip deviation, $\mathrm{cm}$} & ST & 2.0 & 0.1 & $P=0.26$ & $P=0.18$ & $P=0.81$ \\
\hline & DT & 2.0 & 0.1 & & & \\
\hline
\end{tabular}

Response times significantly increased in the dual-task (DT) condition and were also affected by the magnitude of the delay. Gait characteristics such as the stride time and step length significantly depended on the temporal delay, reflecting the systematic gait synchronization. Cognitive loading did not have a significant effect on most of the gait characteristics, as the treadmill enforced a constant walking velocity. ST, single task; CV, coefficient of variation. Significance levels: $* P<0.05, * * * P<0.001$ (in bold).

matic gait changes we observed in the ST condition uniformly, across all delays. Overall, the stride time deviations and the sinusoidal model we report here illustrate that participants significantly and systematically adjusted their stride time in order to synchronize their gait to that of the visual feedback. The introduction of a secondary, cognitive task significantly suppressed this gait synchronization as illustrated by the amplitude of the sinusoid, which was halved.

\section{DISCUSSION}

The present results demonstrate distinct effects of bottom-up visuomotor conflicts as well as top-down cognitive loading on gait agency and gait movements. While the former depended on the spatial and temporal congruency of the feedback, the latter was solely driven by its temporal characteristics as participants attempted to synchronize their gait with the avatar. Cognitive loading enhanced this dissociation: it suppressed gait synchronization across all tested delays but only affected gait agency for selective temporal delays, depending on the participant's step cycle.

\section{Neural Correlates of Agency and the Locomotor Network}

Imaging studies have revealed a widespread network underlying agency processing containing a sensorimotor component, i.e., supplementary motor areas (pre-SMA and SMA), ventral premotor cortex (PMC) as well as the cerebellum (CB), but also posterior parietal cortex (PPC), temporo-parietal junction (TPJ), extrastriate body area, insula, anterior cingulate (ACC), and dorso-lateral prefrontal cortex (PFC) (see review by David et al. 2008). As the PFC, along with TPJ, SMA, PMC, and $\mathrm{ACC}$, has been linked to increased activation during the perception of spatiotemporal sensorimotor conflicts (Farrer et al. 2003; Fink et al. 1999; and error monitoring in general e.g., Ide and $\mathrm{Li}$ 2011), and would additionally be burdened by the secondary task (Miller and Cohen 2001), we predicted an effect of cognitive loading on these components of the agency network.

Human locomotion is controlled by a hierarchical supraspinal locomotor network that includes the frontal cortex, basal ganglia, and brain stem (Nutt et al. 2011). Cortical regions including PFC, SMA, and PMC, overlapping with the agency network described above and likely to be affected by the dual task, have also been identified to control volitional aspects of locomotion such as gait initiation, termination, and changes in direction or velocity during treadmill walking (Gwin et al. 2011; Jahn et al. 2008a; Miyai et al. 2001; Suzuki et al. 2004). Our original hypothesis therefore was that locomotion would be impaired by taxing cognitive resources but, owing to the supervisory role of PFC (see Yogev-Seligmann et al. 2008), more generally and independent of delay.

\section{Gait Agency Is Tuned to Stride Time and Depends on Spatiotemporal Congruency}

The temporal thresholds for gait agency in the present study were $\approx 210 \mathrm{~ms}$ (including the intrinsic delay) and comparable to those previously reported for body part movements (e.g., Farrer et al. 2003) and auditory gait agency (Menzer et al. 2010), a paradigm in which participants judged their sense of agency for temporally delayed sounds of their footfalls. Collectively, the similarity of agency thresholds across a range of studies and protocols suggests that the spatiotemporal limits of agency are determined by a general neural mechanism that is independent of the type of movement and effector. As these studies have provided feedback of action consequences through different sensory modalities (visual or auditory), and because agency thresholds are far above the temporal resolution of the visual (Exner 1875; Sweet 1953) and auditory (Hirsh 1959; Hirsh and Sherrick 1961; cited in Knoblich and Repp 2009) systems, these data additionally promote the idea that agency relies on common supramodal mechanisms, for both goal-directed and continuous movements.

As we did not track participants' eye movements in the present experiment, we cannot entirely exclude that participants glanced at individual body parts during the trials instead of remaining focused on the fixation cross. However, differences in eye fixation cannot account for the observed results. First and as noted above, the temporal thresholds we report are significantly higher than the temporal acuity of the visual 
system and, second, in line with previous literature on the sense of agency, indicating that participants performed an agency task as opposed to a simpler mismatch detection task. Third, it would be difficult, on the basis of eye fixation patterns, to account for the robust main effects of Task or Delay or the interaction between both factors.

Our results suggest that agency may also depend on the particular type of movement as indicated by the cyclic modulations we describe for gait agency. In addition to mechanisms leading to gradual changes in gait agency, agency thus depends on periodic changes of the participant's gait cycle. The ushaped pattern in the agency judgments suggests that the cyclic nature of walking (Blanc et al. 1999; Murray 1967) interfered with gait agency. As such periodicity depends on the walking speed but also the type of movement, e.g., upper limb movements such as finger tapping and full-body movements such as locomotion (Macellari et al. 1999), it is likely that these cycle-dependent agency effects vary for different movements. Furthermore, specific aspects of treadmill walking, such as the fixed velocity and optic flow, differ from free level-ground locomotion. Although temporal gait parameters and kinematics do not appear to change significantly, some joint moments and muscle activations do change (Lee and Hidler 2008). Accordingly, gait agency for treadmill walking will slightly differ from gait agency for level-ground walking.

To the best of our knowledge, no comparable data exist for upper limb movements, as the only studies investigating continuous movement (e.g., circle drawing) were still goal directed (Knoblich and Kircher 2004) or did not investigate the sense of agency (Repp 2011). Leube et al. (2003b) used temporal delays to investigate agency for continuous hand movements but not for delays up to and exceeding movement duration. Further research is therefore necessary to test whether movementdependent agency mechanisms depend on the recurring salient features or may be tuned - in the case of gait agency - to the habitual walking speed, similar to biological motion perception (Jacobs et al. 2004), and could potentially be linked to the acquisition of specific motor skills (Casile and Giese 2006).

\section{Dissociation Between Gait Agency and Movements}

Are these subjective changes in gait agency also reflected in gait movements? Even though our participants were not required to adjust their movements in order to complete a trial, we report systematic stride time changes, especially in the ST condition, suggesting that participants attempted to synchronize their own gait to that of their virtual body. Extending previous findings in goal-directed agency (Fourneret and Jeannerod 1998; Franck et al. 2001; Kannape et al. 2010; Nielsen 1963), our findings further point to a strong dissociation between agency and locomotion, in line with a study by Menzer et al. (2010) in which participants systematically changed their stride time in response to variedly delayed auditory feedback of their footfalls. Thus in HD trials gait movements showed minor changes analogously to ND and FD trials, whereas gait agency changes were different and massively altered (participants judged the vast majority of trials not to be self-generated, opposite to their ND/FD responses). In goal-directed upper limb studies, such dissociations have not been reported and the more participants were required to adjust their movements in order to reach the target, the lower their corresponding agency ratings became (Fourneret et al. 2002; Knoblich and Kircher 2004; Slachevsky et al. 2001). As our task did not require participants to adjust their gait, we suggest that the temporal information of the feedback was automatically integrated into forward motor planning, a property of locomotion often employed in neurorehabilitation (rhythmic auditory stimulation; Hayden et al. 2009; Thaut et al. 1997) and in line with recent studies on side-by-side walkers (Nessler and Gilliland 2009; Zivotofsky and Hausdorff 2007). We argue that the visuospatial incongruence (left-right reversal) in our study may have outweighed the temporal synchrony for gait agency judgments, creating the strong dissociation between agency and gait. We note that these findings cannot be explained by short- or long-term gait adaptation (Choi and Bastian 2007; Gordon et al. 1995; Jensen et al. 1998; Pearson 2000), as all delays were randomized across trials and each trial lasted just 3 s. Furthermore, our participants changed their stride time even though this was not required in order to maintain balance (as in split-belt or circular treadmill studies) and were unaware of their changes in gait (post hoc interviews), in line with evidence that self-initiated gait changes can occur outside awareness (Varraine et al. 2002).

While a comparator mechanism explains the changes observed for sensorimotor control, i.e., an integration of the delay resulting in synchronization (Wolpert et al. 1995), the present gait agency data illustrate that the same comparator may not underlie action attribution at all times as proposed by the central monitoring framework (Blakemore et al. 2002). Instead, these data are compatible with the view that an overt action, in this case gait synchronization, may not have a covert counterpart, i.e., an internal representation of the goal, and point to separate pathways that distinguish between "what" an action is about and "who" the agent is (Georgieff and Jeannerod 1998). This dissociation becomes especially apparent in the HD trials. The spatial information in the feedback, i.e., left-right reversal, may be salient enough to correctly dismiss the feedback without disentangling the temporal sensorimotor conflict.

\section{Cognitive Effects on Gait Agency and Movements}

One main motivation for our study was to investigate the role of cognition in agency. We argue that the introduction of a cognitive task impaired a central comparator mechanism involved in sensorimotor control, leading to the selective effect on agency attribution. Current neuro-cognitive models of the sense of agency (Blakemore et al. 2002; Synofzik et al. 2008a) rely on models of sensorimotor control (Wolpert et al. 1995), neither of which takes the availability of cognitive resources into account. However, the effects of taxing cognitive resources on human locomotion are well described: dual tasking may alter body posture as well as gait characteristics such as velocity, stride variability, and stride asymmetry and lead to a freezing of gait (Woollacott and Shumway-Cook 2002; YogevSeligmann et al. 2008). To the best of our knowledge no data currently exist that describe how agency processing, which relies on sensorimotor comparator mechanisms, is affected by dual tasking.

In the DT condition, participants no longer synchronized their gait to that of their avatar. As the agency task was identical between the two experimental blocks, this implies 
that the difference in motor behavior was induced by the additional cognitive task. In principle, the suppression of gait synchronization may result from an impaired forward model (estimation of future state), noisier sensory feedback, an impaired comparator mechanism (between current and estimated state), or a combination of any or all of these factors. Alternatively, it may be an attentional effect. If cognitive loading introduced noise to the forward model, the output of the comparator should be impaired across all delays and result in delay-independent changes in both sensorimotor control and agency attribution. As our results illustrate, the latter is not the case. Agency processing is unaffected in ND and FD trials. Similarly, agency attribution would be generally affected by the secondary task, if the sensory feedback was altered or this was a main effect of attention. Again, this is not the case. Instead, the selective effect of cognitive loading on agency processing, in combination with specific delays between 225 and $975 \mathrm{~ms}$, suggests that the change occurs at the level of the comparator. In ND and FD trials, the error signal generated by the comparator remains low, as feedback is (pseudo)synchronous to the ongoing movement. Stride time is not changed, and the cognitive load does not affect agency attribution. However, in trials with 225- to 975-ms delay, the comparator fails to generate a sufficient error signal. Further research is required to elucidate whether this inadequate error generation is a direct result of the cognitive load or related to an interaction with an increasing competition for attention between the agency task and the simultaneous arithmetic task. In either case, the limitation prevents gait synchronization and leads to higher selfattribution; this resembles data from schizophrenic patients, in whom overattribution has been linked to an increased temporal binding and an "unusually tolerant comparator" (Daprati et al. 1997; Franck et al. 2001; Haggard et al. 2003).

In the present paradigm one could argue that participants are in fact performing three tasks in parallel: first, the motor task of walking on the treadmill; second, the dual task of backwards counting; third, the agency task requiring participants to monitor the visual feedback of their own movement. Our results illustrate that the interplay between these (three) tasks is crucial with respect to the perceived sense of agency. Independent of whether or not this is regarded as double or triple tasking, the reported effects crucially depend on the introduction of the arithmetic task as hypothesized at the outset and illustrate the possibility of combining such tasks in a controlled, experimental manipulation.

\section{Whole Body Representations}

Locomotion differs from arm movements in stable or sitting actors as it gives rise to vestibular sensations as well as visual changes concerning the surrounding extrapersonal space, making it of relevance for a fundamental aspect of bodily selfconsciousness (Jeannerod 2007), namely, that the self is experienced as a single coherent representation of the entire, spatially situated body (Blanke and Metzinger 2009). Recent experimental procedures have illustrated the multisensory mechanisms underlying the related sense of ownership for the full body (Ehrsson 2007; Ionta et al. 2011; Lenggenhager et al. 2007; Petkova et al. 2011), extending earlier data on body part ownership (Botvinick and Cohen 1998; Dieguez et al. 2009; Lenggenhager et al. 2007) and emphasizing the importance of whole body representations for human self-consciousness (Blanke 2012). Although this was not directly tested here, the present data and the work of Kannape et al. (2010) suggest that, akin to full-body ownership, agency for movements of the entire body addresses a single coherent body representation, similar to that of an individual limb, that is dynamically malleable. While our present data do not categorically distinguish between full-body agency for locomotion and, e.g., body part agency for a single leg, as there is no direct comparison, we argue in favor of the former based on the changing relationship between agency, gait, and cognitive load. The described interactions support the notion that agency attribution may not depend on a single aspect or body part during locomotion, as this interaction can only be explained if participants performed a full-body agency task that was selectively affected by a visual spatiotemporal mismatch and the concurrent cognitive load.

\section{Conclusion}

In conclusion, our results illustrate for the first time the role of cognition and the distinct effects of cognitive loading on gait agency and gait movements. Although partly compatible with a common supramodal mechanism, both for goal-directed and continuous movements, the present findings further provide novel evidence that the neural mechanisms underlying the sense of agency vary depending on the type of action and demands of a task (see also Leube et al. 2003a). We found that in ND and FD trials the common comparator network may suffice to correctly self-attribute gait. Delaying the feedback, however, adds a sensorimotor mismatch and may engage additional cortical resources (likely including PFC) to resolve this conflict. This is enhanced in the DT condition, where this network is involved in three tasks: the arithmetic of the secondary task, resolving the temporal conflict for agency processing, and driving locomotor synchronization.

The selective effects of experimentally induced taxing of cognitive resources resemble a deficit in central monitoring (Frith 2005; Frith et al. 2000; Jeannerod 2009) previously hypothesized to underlie an abnormal sense of agency in schizophrenic populations (Fourneret et al. 2002; Franck et al. 2001). These selective effects contrast with the generally inhibiting effect of dual tasking on gait movements. As illustrated by the sinusoidal model and the effective stride time deviation, cognitive loading suppressed the gait synchronization we observed in the ST condition across all delays. The present paradigm may therefore provide an interesting research tool for investigating both agency and locomotion under cognitive constraints in healthy populations, patients with difficulties in agency attribution, as well as patients with neurodegenerative diseases afflicting sensorimotor and volitional control of locomotion such as Parkinson's disease (Hausdorff et al. 2003; Yogev et al. 2005).

\section{GRANTS}

The authors are supported by grants from the Swiss National Science Foundation (SINERGIA CRSII1-125135), the European Science Foundation (FP7 project VERE), and the Bertarelli Foundation.

\section{DISCLOSURES}

No conflicts of interest, financial or otherwise, are declared by the author(s). 


\section{AUTHOR CONTRIBUTIONS}

Author contributions: O.A.K. and O.B. conception and design of research; O.A.K. performed experiments; O.A.K. analyzed data; O.A.K. and O.B. interpreted results of experiments; O.A.K. prepared figures; O.A.K. drafted manuscript; O.A.K. and O.B. edited and revised manuscript; O.A.K. and O.B. approved final version of manuscript.

\section{REFERENCES}

Armstrong DM. The supraspinal control of mammalian locomotion. J Physiol 405: 1-37, 1988.

Blake R, Shiffrar M. Perception of human motion. Annu Rev Psychol 58: 47-73, 2007.

Blakemore SJ, Wolpert DM, Frith CD. Abnormalities in the awareness of action. Trends Cogn Sci 6: 237-242, 2002.

Blanc Y, Balmer C, Landis T, Vingerhoets F. Temporal parameters and patterns of the foot roll over during walking: normative data for healthy adults. Gait Posture 10: 97-108, 1999.

Blanke O. Multisensory brain mechanisms of bodily self-consciousness. Nat Rev Neurosci 13: 556-571, 2012.

Blanke O, Metzinger T. Full-body illusions and minimal phenomenal selfhood. Trends Cogn Sci 13: 7-13, 2009.

Botvinick M, Cohen J. Rubber hands "feel" touch that eyes see. Nature 391: 756, 1998.

Burnham KP. Multimodel inference: understanding AIC and BIC in model selection. Sociol Methods Res 33: 44, 2004.

Casile A, Giese MA. Nonvisual motor training influences biological motion perception. Curr Biol 16: 69-74, 2006.

Castiello U. The neuroscience of grasping. Nat Rev Neurosci 6: 726-736, 2005 .

Choi JT, Bastian AJ. Adaptation reveals independent control networks for human walking. Nat Neurosci 10: 1055-1062, 2007.

Daprati E, Franck N, Georgieff N, Proust J, Pacherie E, Dalery J, Jeannerod M. Looking for the agent: an investigation into consciousness of action and self-consciousness in schizophrenic patients. Cognition 65: 7186, 1997.

Daprati E, Sirigu A. Laterality effects on motor awareness. Neuropsychologia 40: 1379-1386, 2002.

David N, Bewernick BH, Cohen MX, Newen A, Lux S, Fink GR, Shah NJ, Vogeley K. Neural representations of self versus other: visual-spatial perspective taking and agency in a virtual ball-tossing game. J Cogn Neurosci 18: 898-910, 2006.

David N, Newen A, Vogeley K. The "sense of agency" and its underlying cognitive and neural mechanisms. Conscious Cogn 17: 523-534, 2008.

Dieguez S, Mercier MR, Newby N, Blanke O. Feeling numbness for someone else's finger. Curr Biol 19: R1108-R1109, 2009.

Dietz V, Colombo G, Jensen L. Locomotor activity in spinal man. Lancet 344: 1260-1263, 1994.

Ehrsson HH. The experimental induction of out-of-body experiences. Science 317: 1048, 2007

Exner S. Experimentelle Untersuchungen Der Einfachsten Psychischen Processe. III. Der Persönlichen Gleichung Zweiter Theil. Pflügers Arch 11: 33, 1875.

Farrer C, Franck N, Georgieff N, Frith CD, Decety J, Jeannerod M. Modulating the experience of agency: a positron emission tomography study. Neuroimage 18: 324-333, 2003.

Fink GR, Marshall JC, Halligan PW, Frith CD, Driver J, Frackowiak RS, Dolan RJ. The neural consequences of conflict between intention and the senses. Brain 122: 497-512, 1999.

Fourneret P, de Vignemont F, Franck N, Slachevsky A, Dubois B, Jeannerod M. Perception of self-generated action in schizophrenia. Cogn Neuropsychiatry 7: 139-156, 2002.

Fourneret $\mathbf{P}$, Jeannerod M. Limited conscious monitoring of motor performance in normal subjects. Neuropsychologia 36: 1133-1140, 1998.

Franck N, Farrer C, Georgieff N, Marie-Cardine M, Dalery J, d'Amato T, Jeannerod M. Defective recognition of one's own actions in patients with schizophrenia. Am J Psychiatry 158: 454-459, 2001.

Frith C. The self in action: lessons from delusions of control. Conscious Cogn 14: 752-770, 2005.

Frith CD, Blakemore SJ, Wolpert DM. Abnormalities in the awareness and control of action. Philos Trans R Soc Lond B Biol Sci 355: 1771-1788, 2000.

Frith CD, Done DJ. Experiences of alien control in schizophrenia reflect a disorder in the central monitoring of action. Psychol Med 19: 359-363, 1989.
Georgieff N, Jeannerod M. Beyond consciousness of external reality: a "who" system for consciousness of action and self-consciousness. Conscious Cogn 7: 465-477, 1998.

Geyer H, Herr H. A muscle-reflex model that encodes principles of legged mechanics produces human walking dynamics and muscle activities. IEEE Trans Neural Syst Rehabil Eng 18: 263-273, 2010.

Gordon CR, Fletcher WA, Melvill Jones G, Block EW. Adaptive plasticity in the control of locomotor trajectory. Exp Brain Res 102: 540-545, 1995.

Grillner S, Wallen P. Central pattern generators for locomotion, with special reference to vertebrates. Аnпи Rev Neurosci 8: 233-261, 1985.

Gwin JT, Gramann K, Makeig S, Ferris DP. Electrocortical activity is coupled to gait cycle phase during treadmill walking. Neuroimage 54: 1289-1296, 2011.

Haggard P, Chambon V. Sense of agency. Curr Biol 22: R390-R392, 2012. Haggard P, Martin F, Taylor-Clarke M, Jeannerod M, Franck N. Awareness of action in schizophrenia. Neuroreport 14: 1081-1085, 2003.

Hausdorff JM, Balash J, Giladi N. Effects of cognitive challenge on gait variability in patients with Parkinson's disease. J Geriatr Psychiatry Neurol 16: 53-58, 2003

Hayden R, Clair AA, Johnson G, Otto D. The effect of rhythmic auditory stimulation (RAS) on physical therapy outcomes for patients in gait training following stroke: a feasibility study. Int J Neurosci 119: 2183-2195, 2009.

Helmholtz H. Handbuch der Physiologischen Optik. Leipzig, Germany: Voss, 1866.

Hirsh IJ. Auditory perception of temporal order. J Acoust Soc Am 31: 9, 1959.

Hirsh IJ, Sherrick CE Jr. Perceived order in different sense modalities. J Exp Psychol 62: 423-432, 1961.

Ide JS, Li CS. A cerebellar thalamic cortical circuit for error-related cognitive control. Neuroimage 54: 455-464, 2011.

Ionta S, Heydrich L, Lenggenhager B, Mouthon M, Fornari E, Chapuis D, Gassert R, Blanke O. Multisensory mechanisms in temporo-parietal cortex support self-location and first-person perspective. Neuron 70: 363-374, 2011.

Jacobs A, Pinto J, Shiffrar M. Experience, context, and the visual perception of human movement. J Exp Psychol Hum Percept Perform 30: 822-835, 2004.

Jahn K, Deutschlander A, Stephan T, Kalla R, Hufner K, Wagner J, Strupp M, Brandt T. Supraspinal locomotor control in quadrupeds and humans. Prog Brain Res 171: 353-362, 2008a.

Jahn K, Deutschlander A, Stephan T, Kalla R, Wiesmann M, Strupp M, Brandt T. Imaging human supraspinal locomotor centers in brainstem and cerebellum. Neuroimage 39: 786-792, 2008b.

Jeannerod M. Being oneself. J Physiol (Paris) 101: 161-168, 2007.

Jeannerod M. The mechanism of self-recognition in humans. Behav Brain Res 142: 1-15, 2003.

Jeannerod M. Motor Cognition: What Actions Tell the Self. Oxford, UK: Oxford Univ. Press, 2006, p. 224.

Jeannerod M. The sense of agency and its disturbances in schizophrenia: a reappraisal. Exp Brain Res 192: 527-532, 2009.

Jeannerod M, Pacherie E. Agency, simulation and self-identification. Mind Lang 19: 113-146, 2004.

Jensen L, Prokop T, Dietz V. Adaptational effects during human split-belt walking: influence of afferent input. Exp Brain Res 118: 126-130, 1998.

Kandel ER. Principles of Neural Science. New York: McGraw-Hill, 2012.

Kannape OA, Blanke O. Agency, gait and self-consciousness. Int J Psychophysiol 83: 191-199, 2012.

Kannape OA, Schwabe L, Tadi T, Blanke O. The limits of agency in walking humans. Neuropsychologia 48: 1628-1636, 2010.

Knoblich G, Kircher TT. Deceiving oneself about being in control: conscious detection of changes in visuomotor coupling. J Exp Psychol Hum Percept Perform 30: 657-666, 2004.

Knoblich G, Repp BH. Inferring agency from sound. Cognition 111: 248 262, 2009.

Knoblich G, Stottmeister F, Kircher T. Self-monitoring in patients with schizophrenia. Psychol Med 34: 1561-1569, 2004.

Lee SJ, Hidler J. Biomechanics of overground vs. treadmill walking in healthy individuals. J Appl Physiol 104: 747-755, 2008.

Lenggenhager B, Tadi T, Metzinger T, Blanke O. Video ergo sum: manipulating bodily self-consciousness. Science 317: 1096-1099, 2007.

Leube DT, Knoblich G, Erb M, Grodd W, Bartels M, Kircher TT. The neural correlates of perceiving one's own movements. Neuroimage 20: 2084-2090, 2003a. 
Leube DT, Knoblich G, Erb M, Kircher TT. Observing one's hand become anarchic: an fMRI study of action identification. Conscious Cogn 12: 597-608, 2003b.

Macellari V, Giacomozzi C, Saggini R. Spatial-temporal parameters of gait: reference data and a statistical method for normality assessment. Gait Posture 10: 171-181, 1999.

Markowitz J, Krishnaswamy P, Eilenberg MF, Endo K, Barnhart C, Herr H. Speed adaptation in a powered transtibial prosthesis controlled with a neuromuscular model. Philos Trans R Soc Lond B Biol Sci 366: 1621-1631, 2011.

Menzer F, Brooks A, Halje P, Faller C, Vetterli M, Blanke O. Feeling in control of your footsteps: conscious gait monitoring and the auditory consequences of footsteps. Cogn Neurosci 1: 184-192, 2010.

Miller EK, Cohen JD. An integrative theory of prefrontal cortex function. Annu Rev Neurosci 24: 167-202, 2001.

Miyai I, Tanabe HC, Sase I, Eda H, Oda I, Konishi I, Tsunazawa Y, Suzuki T, Yanagida T, Kubota K. Cortical mapping of gait in humans: a near-infrared spectroscopic topography study. Neuroimage 14: 1186-1192, 2001

Murray MP. Gait as a total pattern of movement. Am J Phys Med 46: 290-333, 1967.

Nessler JA, Gilliland SJ. Interpersonal synchronization during side by side treadmill walking is influenced by leg length differential and altered sensory feedback. Hum Mov Sci 28: 772-785, 2009.

Nielsen T. Volition: a new experimental approach. Scand J Psychol 4: 225-230, 1963.

Noble JW, Prentice SD. Adaptation to unilateral change in lower limb mechanical properties during human walking. Exp Brain Res 169: 482-495, 2006.

Nutt JG, Horak FB, Bloem BR. Milestones in gait, balance, and falling. Mov Disord 26: 1166-1174, 2011.

Pearson KG. Neural adaptation in the generation of rhythmic behavior. Annu Rev Physiol 62: 723-753, 2000.

Perret C, Cabelguen JM. Main characteristics of the hindlimb locomotor cycle in the decorticate cat with special reference to bifunctional muscles. Brain Res 187: 333-352, 1980.

Petkova VI, Bjornsdotter M, Gentile G, Jonsson T, Li TQ, Ehrsson HH. From part- to whole-body ownership in the multisensory brain. Curr Biol 21: $1118-1122,2011$.

R Development Core Team. $R$ : A Language and Environment for Statistical Computing. Vienna: R Foundation for Statistical Computing, 2011.

Repp BH. Comfortable synchronization of cyclic drawing movements with a metronome. Hum Mov Sci 30: 18-39, 2011.

Repp BH. Does an auditory distractor sequence affect self-paced tapping? Acta Psychol (Amst) 121: 81-107, 2006.

Repp BH. Sensorimotor synchronization: a review of the tapping literature. Psychon Bull Rev 12: 969-992, 2005.

Repp BH, Knoblich G. Toward a psychophysics of agency: detecting gain and loss of control over auditory action effects. J Exp Psychol Hum Percept Perform 33: 469-482, 2007.

Rodríguez CC. The ABC of model selection: AIC, BIC and the new CIC. AIP Conf Proc 803: 80-87, 2005.

Shik ML, Orlovsky GN. Neurophysiology of locomotor automatism. Physiol Rev 56: 465-501, 1976
Slachevsky A, Pillon B, Fourneret P, Pradat-Diehl P, Jeannerod M, Dubois B. Preserved adjustment but impaired awareness in a sensory-motor conflict following prefrontal lesions. J Cogn Neurosci 13: 332-340, 2001.

Sperry RW. Neural basis of the spontaneous optokinetic response produced by visual inversion. J Comp Physiol Psychol 43: 482-489, 1950.

Springer S, Giladi N, Peretz C, Yogev G, Simon ES, Hausdorff JM. Dual-tasking effects on gait variability: the role of aging, falls, and executive function. Mov Disord 21: 950-957, 2006.

Suzuki M, Miyai I, Ono T, Oda I, Konishi I, Kochiyama T, Kubota K. Prefrontal and premotor cortices are involved in adapting walking and running speed on the treadmill: an optical imaging study. Neuroimage 23: 1020-1026, 2004.

Sweet AL. Temporal discrimination by the human eye. Am J Psychol 66: 185-198, 1953.

Synofzik M, Thier P, Lindner A. Internalizing agency of self-action: perception of one's own hand movements depends on an adaptable prediction about the sensory action outcome. J Neurophysiol 96: 1592-1601, 2006.

Synofzik M, Vosgerau G, Newen A. Beyond the comparator model: a multifactorial two-step account of agency. Conscious Cogn 17: 219-239, 2008 a.

Synofzik M, Vosgerau G, Newen A. I move, therefore I am: a new theoretical framework to investigate agency and ownership. Conscious Cogn 17: 411-424, 2008b.

Thaut MH, McIntosh GC, Rice RR. Rhythmic facilitation of gait training in hemiparetic stroke rehabilitation. J Neurol Sci 151: 207-212, 1997.

Todorov E, Jordan MI. Optimal feedback control as a theory of motor coordination. Nat Neurosci 5: 1226-1235, 2002.

Torres-Oviedo G, Bastian AJ. Seeing is believing: effects of visual contextual cues on learning and transfer of locomotor adaptation. J Neurosci 30: 17015-17022, 2010.

van den Bos E, Jeannerod M. Sense of body and sense of action both contribute to self-recognition. Cognition 85: 177-187, 2002.

van Iersel MB, Ribbers H, Munneke M, Borm GF, Rikkert MG. The effect of cognitive dual tasks on balance during walking in physically fit elderly people. Arch Phys Med Rehabil 88: 187-191, 2007.

Varraine E, Bonnard M, Pailhous J. Interaction between different sensory cues in the control of human gait. Exp Brain Res 142: 374-384, 2002.

von Holst E, Mittelstaedt H. Das Reafferenzprinzip. Naturwissenschaften 20: 13,1950 .

Wichmann FA, Hill NJ. The psychometric function: I. Fitting, sampling, and goodness of fit. Percept Psychophys 63: 1293-1313, 2001a.

Wichmann FA, Hill NJ. The psychometric function: II. Bootstrap-based confidence intervals and sampling. Percept Psychophys 63: 1314-1329, $2001 b$.

Wolpert DM, Ghahramani Z, Jordan MI. An internal model for sensorimotor integration. Science 269: 1880-1882, 1995.

Woollacott M, Shumway-Cook A. Attention and the control of posture and gait: a review of an emerging area of research. Gait Posture 16: 1-14, 2002.

Yogev G, Giladi N, Peretz C, Springer S, Simon ES, Hausdorff JM. Dual tasking, gait rhythmicity, and Parkinson's disease: which aspects of gait are attention demanding? Eur J Neurosci 22: 1248-1256, 2005.

Yogev-Seligmann G, Hausdorff JM, Giladi N. The role of executive function and attention in gait. Mov Disord 23: 329-342, 2008.

Zivotofsky AZ, Hausdorff JM. The sensory feedback mechanisms enabling couples to walk synchronously: an initial investigation. J Neuroeng Rehabil 4: 28, 2007. 